\title{
Neuer Folder für Allergie-Patienten
}

\begin{abstract}
- Die sublinguale spezifische Immuntherapie (SLIT) hat mittlerweile einen festen Platz im Spektrum der Allergietherapien. Wesentliche Grundvoraussetzung für den Behandlungserfolg ist die tägliche Einnahme eines hochdosierten wirksamen und verträglichen AllergenPräparates. Damit Patienten die Anwendung über die empfohlenen drei Jahre auch wirklich beibehalten, ist zum einen ein intensives Aufklä-
\end{abstract}

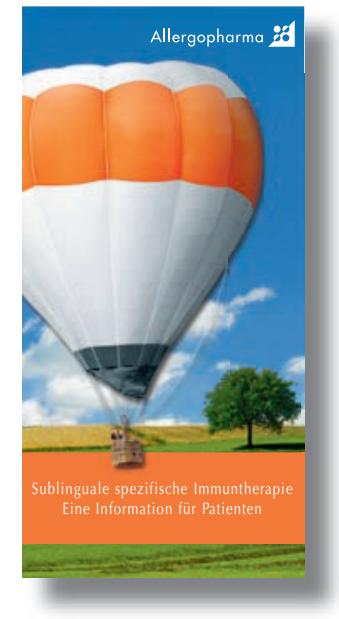

rungsgespräch zu empfehlen. Zum anderen hat es sich als hilfreich und compliancefördernd erwiesen, wenn Arzt und Patient die Therapie „vereinbaren“. Wird diese Vereinbarung auch noch schriftlich festgehalten, erhöht sich die Verbindlichkeit und damit auch die Therapietreue.

Unterstützung für die Bemühungen um die Compliance bietet der neue Patientenfolder „Sublinguale spezifische Immuntherapie" von Aller- gopharma. Er ergänzt das allergologische Beratungsgespräch, liefert Hintergrundinformationen zur Aufdosierung und Anwendung der SLIT und gibt Tipps für den Alltag. Zudem enthält er eine Einverständniserklärung für Arzt und Patient zur Dokumentation des Aufklärungsgesprächs und zum Einverständnis des Patienten mit der Therapie.

Der Folder kann bei Allergopharma unter der Faxnummer 040/72765-268 bestellt werden.

gz

Nach Informationen von Allergopharma

\section{Neues Cochlea-Implantat vorgestellt}

- Concerto $^{\mathrm{TM}}$ heißt das neue Cochlea-Implantat (CI), das der Hersteller MED-EL Medical Electronics kürzlich auf der Leitkonferenz $\mathrm{Cl} 2010$ in Stockholm vorstellte. Es gehört zur Maestro 2010, der neuen Generation von Cochlea-Implantatsystemen von MED-EL.

Das Herzstück des Systems, das CI Concerto $^{\text {TM }}$, ist um 25 Prozent dünner als das Vorgängermodell und eignet sich deshalb noch besser für minimal-invasive Operationstechniken. Das zweite Element des Systems bildet der ebenfalls neu entwickelte, hinter dem Ohr getragene Audioprozessor OPUS 2. Mit der um bis zu 50\% längeren Batterielebensdauer besitzt er eine durchgehende Nutzungsdauer von bis zu 90 Stunden. Neue Farben, darunter auch vier neue Designs speziell für Kinder, stehen zur Auswahl. Auch der dritte Baustein des
Systems, die MAESTRO System Software 4.0, wurde neu entwickelt. Sie verfügt über 40 neue Funktionen. Mit der modernisierten Software erhalten Audiologen und CI-Experten eine optimale Unterstützung für eine flexiblere und effizientere Anpassung der Produkte an die persönlichen Bedürfnisse des Nutzers.

gz

Nach Informationen von MED-EL

\section{Mehr Compliance durch Cluster-SIT}

- Die subkutane spezifische Immuntherapie (SCIT) ist bei IgE-vermittelten Allergien die am häufigsten angewendete kausale Therapieform. Bei konventioneller Aufdosierung ist der Therapiestart allerdings für Arzt und Patient mit einigem Zeitaufwand verbunden. Eine schnelle und gut verträgliche Alternative zur konventionellen Aufdosierung ist die Cluster-Therapie, sie ermöglicht das Erreichen der Erhaltungsdosis innerhalb kurzer Zeit.

Mit Clustoid ${ }^{\circledR}$ bietet ROXALL Medizin $\mathrm{GmbH}$ ein Präparat an, das speziell für die Cluster-Immuntherapie entwickelt wurde. Mit einem Hochpolymerisations-Verfahren werden Cluster-Allergoide (Clustoide) hergestellt, die sowohl in der Verträglichkeit als auch in ihrer Wirksamkeit überzeugen. Bereits am ersten Behandlungstag wird mit nur zwei Injektionen die Höchstdosis erreicht. Der Abstand zwischen beiden Injektionen beträgt lediglich 15 Minuten. Dadurch kann der Aufenthalt der Patienten in der Praxis deutlich verkürzt werden. Clustoid ${ }^{\circledR}$ gibt es nur in einer Stärke, was die Handhabung in der Praxis zusätzlich erleichtert. Mit dem Therapiestart muss nicht mehr gewartet werden, bis der Pollenflug vorbei ist, die Aufdosierung kann auch in der Pollensaison erfolgen.

Auf dem diesjährigen Kongress der EAACI (European Academy of Allergolgy and Clinical Immunology) in London wurden Allergologen aus aller Welt über die Produktneuerungen informiert. Der Start in Deutschland erfolgte im Juli 2010 . gz

Nach Informationen von Roxall Medizin

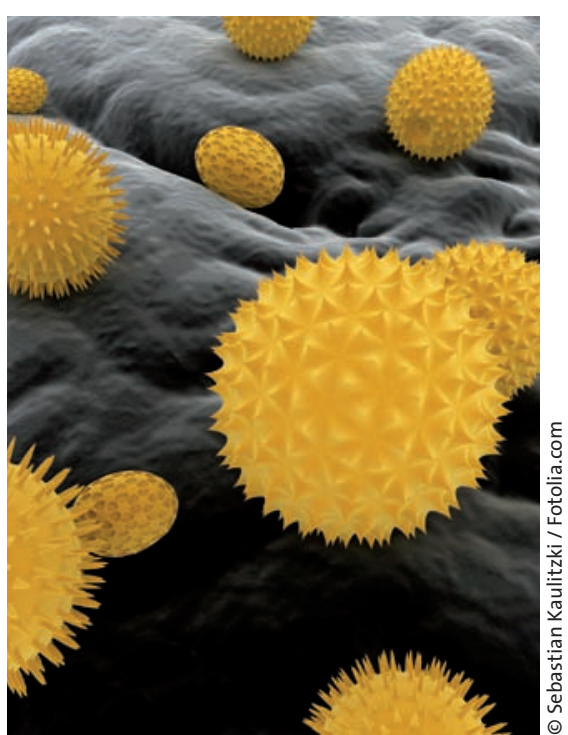

IgE-vermittelten Allergien wie die Pollenallergie lassen sich mit der spezifischen Immuntherapie wirksam bekämpfen. 